\title{
Ingestão de ração e comportamento de larvas de pacu em resposta a estímulos químicos e visuais
}

\author{
Marcelo Borges Tesser ${ }^{1}$, Maria Célia Portella ${ }^{2}$ \\ ${ }^{1}$ Doutor em Aqüicultura - UNESP - Centro de Aqüicultura, Jaboticabal. \\ 2 UNESP - Departamento de Biologia Aplicada à Agropecuária, Jaboticabal.
}

RESUMO - Este estudo foi realizado com o objetivo de comparar a influência dos estímulos visual e/ou químico de náuplios de Artemia e de dieta microencapsulada sobre a taxa de ingestão da dieta microencapusulada por larvas de pacu Piaractus mesopotamicus. Utilizou-se um esquema fatorial 7 x 4 (estímulos e idades) com duas repetições. Verificou-se efeito da idade das larvas e dos estímulos, mas não houve efeito para a interação idade × estímulos. O estímulo químico da Artemia e ambos os estímulos da Artemia resultaram em maior taxa de ingestão de dieta inerte. Resultado intermediário foi obtido com o estímulo visual da dieta microencapsulada. O estímulo químico, em comparação ao estímulo visual da Artemia, resultou em maiores taxas de ingestão da dieta. Com o aumento da idade, houve incremento na taxa de ingestão. Os estímulos visual e químico dos náuplios e o estímulo visual da ração aumentaram a ingestão de dieta inerte por larvas de pacu. Náuplios de Artemia devem ser oferecidos antes do fornecimento da dieta inerte, pois podem auxiliar no processo de transição alimentar. Os resultados deste trabalho apontaram novas possibilidades de estudos com larvas de peixes neotropicais visando a substituição precoce do alimento vivo para o inerte.

Palavras-chave: Artemia, comportamento, estímulo alimentar, larva, pacu, Piaractus mesopotamicus

\section{Diet ingestion rate and pacu larvae behavior in response to chemical and visual stimuli}

\begin{abstract}
The effect of visual, chemical and the combination of both stimuli from Artemia nauplii and from microencapsulated diet on dry diet ingestion by pacu Piaractus mesopotamicus larvae was evaluated in this research. The experiment was analyzed as a $7 \times 4$ factorial arrangement (seven stimuli and four ages) with two replicates. It was observed effect of larvae age and stimuli, but no interaction (age $\times$ stimuli) was observed. The chemical effect from Artemia and both effects from Artemia resulted in higher ingestion rates. An intermediary result was obtained with visual effect from microencapsulated diet. The chemical stimulus from Artemia resulted in higher ingestion rates than that obtained with Artemia visual stimulus. Ingestion rate increased as age increased. Chemical and visual stimuli from nauplii and visual stimuli from diet increased microencapsulated diet ingestion by pacu larvae. Artemia nauplii offered before the artificial diet may assist weaning. These results opens new study possibilities with Neotropical fish larvae precocious weaning.
\end{abstract}

Key Words: Artemia, behavior, feeding stimulus, larvae, pacu, Piaractus mesopotamicus

\section{Introdução}

Um fator decisivo para o sucesso da transição do alimento vivo para o inerte para larvas de peixes é a atratividade do alimento (Kolkovski et al., 2000). O sistema quimiorreceptor em peixes é muito bem desenvolvido e serve para mediar vários comportamentos fundamentais, entre eles, o da procura por alimento (Sorensen \& Caprio, 1997). Além do sistema quimiorreceptor, muitos peixes dependem da visão para procurar o alimento (Guthrie, 1986). Planas \& Cunha (1999) afirmam que um dos maiores problemas na utilização do alimento artificial é a falta de estímulo visual das dietas, pois as larvas ingerem principalmente organismos vivos (que se movimentam) durante os primeiros dias de desenvolvimento.

Kolkovski et al. (1997) estudaram a influência dos estímulos químicos e visuais provenientes de náuplios de Artemia sobre a ingestão de dieta inerte por larvas de Sparus aurata. Neste estudo pioneiro, ficou evidenciada a importância individual desses estímulos e de sua combinação para obtenção de uma taxa de ingestão de alimento inerte até $120 \%$ superior à de larvas tratadas sem nenhum estímulo. Recentemente, Tesser et al. (2005) citaram que a alimentação conjunta de náuplios de Artemia e de uma dieta microencapsulada, possivelmente em virtude dos estímulos visuais e químicos, promoveu maior ingestão da 
dieta por larvas de pacu. No entanto, ainda são necessárias avaliações mais precisas de como e quais estímulos realmente influenciam a ingestão de dieta inerte por larvas desta espécie.

O pacu (Piaractus mesopotamicus) é um peixe de água doce originário da bacia Paraná-Paraguai e apresenta grande potencial para a aqüicultura, pois é uma espécie de hábito alimentar onívoro, rápido crescimento, boa qualidade de carne e boa aceitação pelo mercado consumidor (Jomori, 1999). Segundo a estatística de produção publicada pelo IBAMA (2004), referente ao ano de 2003, o pacu é a segunda espécie nativa mais criada, atrás apenas do tambaqui (Colossoma macropomum), outro representante da família Serrassalminae. Atualmente, a criação inicial de pacu no Brasil é feita em sistema semi-intensivo de produção, que resulta em baixas taxas de sobrevivência. Segundo Bock \& Padovani (2000), após 30 dias de cultivo inicial, a taxa de sobrevivência desta espécie mantém-se em torno de 35 e $50 \%$. No entanto, valores de sobrevivência inferiores têm sido reportados na literatura (Chabalin et al., 1989; Jomori et al., 2003). Uma alternativa promissora para este sistema é a produção intensiva desta espécie em laboratório, que resulta em taxas de sobrevivência superiores às obtidas em sistema semi-intensivo (Jomori et al., 2003). No entanto, a transição no fornecimento do alimento vivo para o inerte ainda representa um entrave para sua produção intensiva. Neste estudo, objetivou-se verificar a influência dos estímulos visual e químico de náuplios de Artemia e de dieta microencapsulada sobre o comportamento e a taxa de ingestão da mesma dieta por larvas de pacu.

\section{Material e Métodos}

As larvas de pacu (Piaractus mesopotamicus) recémeclodidas, provenientes de desova induzida, foram estocadas em tanques de $100 \mathrm{~L}$, com renovação de água e aeração constantes. A água utilizada no experimento, proveniente de poço artesiano, foi mantida com $\mathrm{pH}$ médio de 8,2 e temperatura constante de $29^{\circ} \mathrm{C}$, por meio de aquecedores submersos. As larvas foram alimentadas com náuplios de Artemia até 12 horas antes do início do teste, quando a alimentação foi suspensa e as larvas transferidas para as unidades experimentais.

A coleta de dados foi realizada no $5 \underline{\mathrm{o}}, 9 \underline{\mathrm{o}}, 13 \underline{\mathrm{o}}$ e $17 \underline{\mathrm{o}}$ dias de vida, que corresponderam ao $1 \underline{0}, 5 \underline{0}$, go e $13 \underline{0}$ dias de alimentação exógena. Em cada dia experimental, foi realizada biometria com aproximadamente dez larvas para sua caracterização morfométrica (Tabela 1).

Nesse período, foram realizados os seguintes testes: EQA - estímulo químico proveniente da Artemia; EQDM -
Tabela 1 - Peso $(\mathrm{mg})$ e comprimento total $(\mathrm{mm})$ médios (desviopadrão, $n=10$ ) das larvas de pacu nos diferentes dias experimentais

Table 1 - Mean total weight $(\mathrm{mg})$ and length $(\mathrm{mm}) \pm$ standard deviation $(n=10)$ of pacu larvae at different experimental days

\begin{tabular}{lcc}
\hline $\begin{array}{l}\text { Dias de vida } \\
\text { Days of life }\end{array}$ & $\begin{array}{c}\text { Peso }(\mathrm{mg}) \\
\text { Weight }(\mathrm{mg})\end{array}$ & $\begin{array}{c}\text { Comprimento total }(\mathrm{mm}) \\
\text { Total length }(\mathrm{mm})\end{array}$ \\
\hline 5 & $0,63 \pm 0,24$ & $5,77 \pm 0,14$ \\
9 & $1,87 \pm 0,48$ & $6,90 \pm 0,46$ \\
13 & $6,30 \pm 1,22$ & $8,47 \pm 0,35$ \\
17 & $8,77 \pm 1,95$ & $9,32 \pm 0,66$ \\
\hline
\end{tabular}

estímulo químico gerado pela dieta microencapsulada; EVA - estímulo visual proveniente da Artemia; EQVA estímulos químico e visual da Artemia; EVDM - estímulo visual gerado pela dieta microencapsulada; EQDM - estímulo químico gerado pela dieta microencapsulada; EQVDM - estímulos químico e visual gerados pela dieta microencapsulada; $\mathrm{C}$ (controle) - ausência de qualquer tipo de estímulo.

As unidades experimentais utilizadas foram béqueres de vidro de $100 \mathrm{~mL}$ mantidos dentro de recipientes plásticos de $300 \mathrm{~mL}$. As larvas foram estocadas nas unidades experimentais na proporção de 10 larvas/béquer, conforme ilustrado na Figura 1.

A avaliação do estímulo visual sobre as larvas de pacu foi realizada por meio da adição de náuplios de Artemia (10 náuplios $/ \mathrm{mL}$ ) e/ou de uma dieta microencapsulada na água do recipiente plástico. Para avaliação do estímulo químico, introduziu-se nos béqueres menores (contendo as larvas) a água utilizada para a eclosão dos náuplios ( $20 \mathrm{~g}$ de cistos em $10 \mathrm{~L}$ de água) ou a água previamente preparada contendo resíduos da dieta microencapsulada.

No estudo da associação de ambos os estímulos (visual e químico), foram realizadas as combinações com água de eclosão dos náuplios ou água de resíduo de dieta microencapsulada, com os náuplios e a própria dieta microencapsulada. Um grupo controle foi mantido nas mesmas condições anteriormente descritas, mas sem a presença de estímulos, para determinação da alimentação espontânea pelas larvas. A água de resíduo da dieta microencapsulada foi preparada minutos antes da execução dos testes, por meio da adição de aproximadamente $2 \mathrm{~g}$ de ração a $50 \mathrm{~mL}$ de água. A mistura foi homogeneizada em agitador magnético por 15 minutos e filtrada em papel-filtro. A dieta microencapsulada utilizada nos testes foi a Hatchfry Encapsulon (Argent Labs, EUA), que, segundo o fabricante, possui $50 \%$ de proteína, $12 \%$ de lipídeos, $3 \%$ de fibra, $7 \%$ de cinzas e $6 \%$ de $=$ umidade.

Após 5 minutos de exposição das larvas aos diferentes estímulos, foi introduzida no meio experimentaluma quanti- 


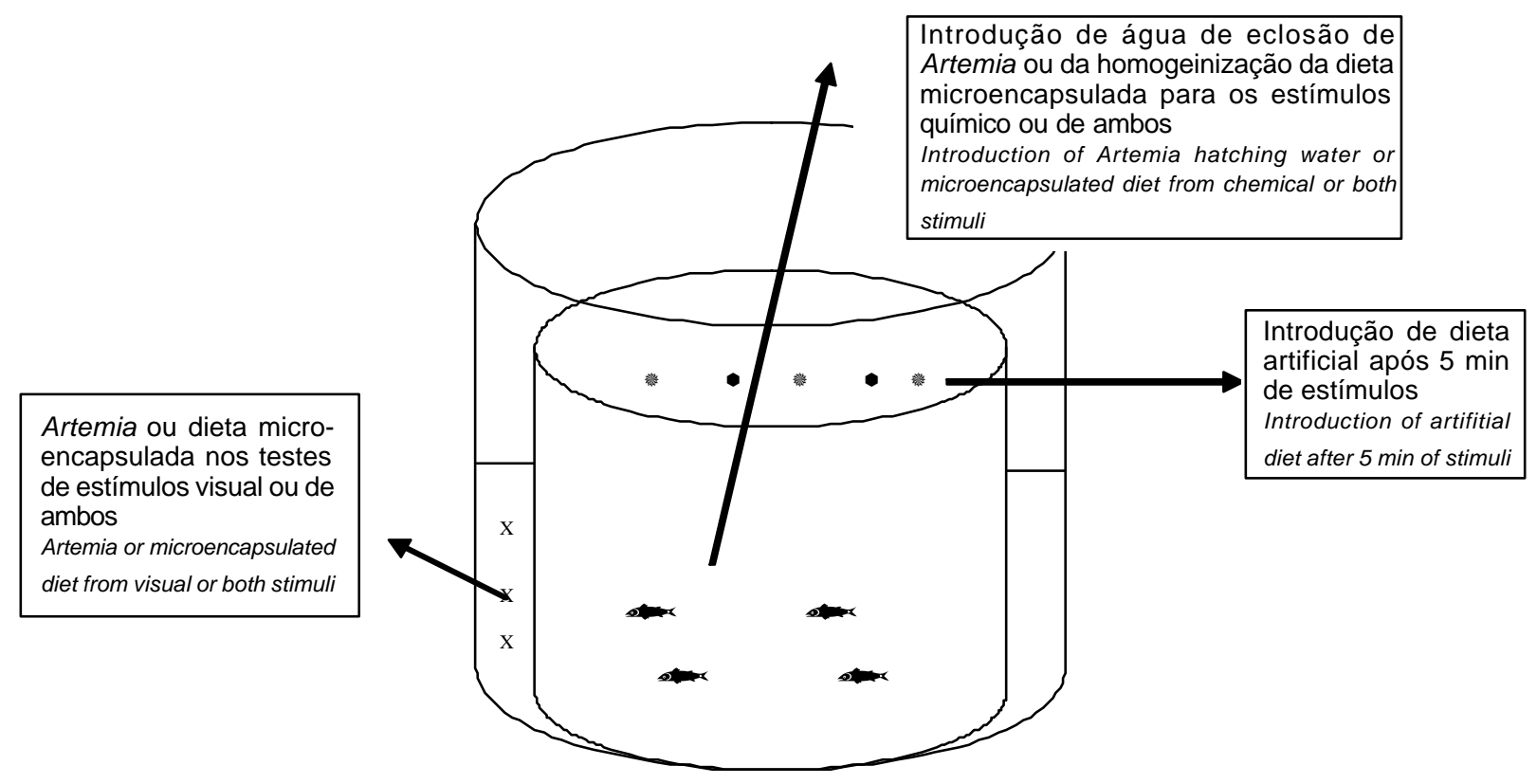

Figura 1 - Esquema experimental utilizado para a avaliação da influência dos estímulos químicos e/ou visuais e da combinação de ambos sobre a taxa de ingestão de dieta microencapsulada por larvas de pacu (Adaptado de Kolkovski et al., 1997).

Figure 1 - Experimental scheme used for evaluation of the effect of chemical, visual and the combination of both stimuli on microencapsulated diet ingestion rate by pacu larvae (Adapted from Kolkovski et al., 1997).

dade em excesso da dieta microencapsulada $(1 \mathrm{~g})$ para as larvas de todos os tratamentos. Dez minutos após a introdução da dieta microencapsulada, as larvas foram coletadas e conservadas em formol $10 \%$ para determinação da taxa de ingestão (Dou et al., 2000), calculada como o número de partículas de alimento por larva. As larvas foram sacrificadas com gelo para prevenir a regurgitação do alimento ingerido. Para verificação da ingestão, o tubo digestivo das dez larvas de cada repetição foi dissecado efetuando-se a contagem dos grânulos de dieta microencapsulada em microscópio estereoscópico.

O comportamento larval foi analisado com base na descrição do comportamento alimentar de larvas de bacalhau, Gadus morhua, determinado por Munk (1995). Neste experimento, observou-se apenas o comportamento de ataque, determinado como um movimento abrupto e curto para frente, concomitantemente à abertura da boca. Foi aplicada a técnica denominada animal focal: três larvas de cada tratamento foram aleatoriamente escolhidas para observação, durante um minuto, do comportamento de captura no período de estímulo inicial e do comportamento durante a introdução da dieta microencapsulada no béquer menor contendo as larvas. Um animal por vez foi observado, diretamente, por apenas um observador.
O experimento foi realizado em delineamento inteiramente casualizado, composto de estímulos e idades, em esquema fatorial 7 x 4 (estímulos e idades), utilizando-se duas repetições por tratamento. Aos valores de taxa de ingestão das larvas de cada tratamento foi aplicada a análise de variância paramétrica (Sokal \& Rohlf, 1995), utilizando-se o programa SAS versão 8.0 (SAS, 1985). Nos resultados em que houve diferença $(\mathrm{P}<0,05)$, as médias foram contrastadas pelo teste Duncan, a $5 \%$ de probabilidade. Para satisfazer as premissas da análise de variância, os resultados foram testados quanto à normalidade (ShapiroWilk) e homogeneidade de variâncias (Levene).

\section{Resultados e Discussão}

Os resultados da taxa de ingestão de dieta microencapsulada obtidos com as larvas de pacu estimuladas por diferentes agentes foram inferiores aos descritos por Cestarolli \& Salles (2000) para a taxa de ingestão de náuplios de Artemia durante o mesmo período de alimentação (10 minutos). A metodologia aplicada neste experimento para determinação da taxa de ingestão foi a mesma utilizada por Dou et al. (2000), que consideraram que as larvas de Paralichthys olivaceus não atingiriam a saciedade após os 10 minutos de exposição ao alimento 
vivo. Esta premissa do teste é muito importante, pois a saciedade nesse ou em menor tempo poderia impedir a detecção de diferenças de consumo entre os tratamentos. Por outro lado, é possível que o período de 10 minutos de exposição das larvas de pacu à dieta inerte tenha sido muito curto para que maior quantidade de grânulos pudesse ser ingerida. No entanto, mesmo com baixa ingestão, foram detectadas diferenças entre os estímulos.

Os resultados da análise (Tabela 2 ) demonstraram que houve efeito $(\mathrm{P}<0,05)$ tanto da idade das larvas quanto dos estímulos sobre a taxa de ingestão, porém, não houve interação desses fatores. Com o aumento da idade das larvas, houve aumento na taxa de ingestão da dieta microencapsulada, de modo que os animais com 9 e 13 dias de vida não diferiram entre si $(\mathrm{P}>0,05)$. Esse resultado difere dos obtidos por Kolkovski et al. (1997), que, por meio do cálculo dos percentuais de incremento da taxa de ingestão em relação ao grupo controle, observaram decréscimo da ingestão com o aumento da idade das larvas de Sparus aurata.

Conforme descrito na Figura 2 e na Tabela 2, verificou-se evidente aumento da taxa de ingestão ao longo do tempo, até mesmo em relação ao grupo controle (C), no qual

Tabela 2 - Valores de F e médias $(n=20)$ para a taxa de ingestão ${ }^{1}$ em larvas de pacu em diferentes idades sob diversos tratamentos

Table 2 - $\quad F$ values and means $(n=20)$ of pacu larvae ingestion rates under different ages and treatments

\begin{tabular}{|c|c|}
\hline $\begin{array}{l}\text { Idade das larvas (dias) } \\
\text { Larval age (days) }\end{array}$ & $\begin{array}{l}\text { Taxa de ingestão } \\
\text { Ingestion rate }\end{array}$ \\
\hline 5 & $0,03 \mathrm{c}$ \\
\hline 9 & $0,94 b$ \\
\hline 13 & $0,87 \mathrm{~b}$ \\
\hline 17 & $2,19 \mathrm{a}$ \\
\hline Estímulo & Taxa de ingestão \\
\hline Stimulus & Ingestion rate \\
\hline Controle $(\mathrm{C})$ & $0,34 \mathrm{c}$ \\
\hline Control $(C)$ & \\
\hline Estímulo visual da Artemia (EVA) & $0,81 \mathrm{bc}$ \\
\hline Visual stimuli from Artemia (EVA) & \\
\hline Estímulo químico da Artemia (EQA) & $1,72 \mathrm{a}$ \\
\hline Chemical stimulus from Artemia ( $E Q A)$ & \\
\hline Estímulos químico e visual da Artemia (EQVA) & $1,61 \mathrm{a}$ \\
\hline Both stimuli from Artemia (EQVA) & \\
\hline Estímulo visual da ração (EVDM) & $1,18 \mathrm{ab}$ \\
\hline Visual stimuli from inert diet (EVDM) & \\
\hline Estímulo químico da ração (EQDM) & $0,94 \mathrm{bc}$ \\
\hline Chemical stimulus from inert diet (EQDM) & \\
\hline Estímulos químico e visual da ração (EQVDM) & $0,47 \mathrm{c}$ \\
\hline Both stimuli from inert diet (EQVDM) & \\
\hline
\end{tabular}

Médias na mesma coluna com letras diferentes são diferentes estatísticamente $(P<0,05)$ pelo teste Duncan.

${ }^{1}$ Número de grânulos ingeridos por larva em 10 minutos.

Means within a column followed by different letters differ $(P<0.05)$ by Duncan test.

${ }^{1}$ Number of ingested granules per larvae in 10 minutes. os níveis foram inferiores aos obtidos nos demais tratamentos. Esse resultado comprova que, até pelo menos 17 dias de vida (12 dias de alimentação exógena), as larvas de pacu respondem positivamente aos estímulos visuais e químicos.

Pouco se sabe sobre o desenvolvimento dos sistemas quiomiorreceptor e visual das larvas de pacu, mas provavelmente houve desenvolvimento dessas estruturas, de modo que a percepção dos estímulos pôde ser incrementada, resultando em maior taxa de ingestão com o tempo. Roo et al. (1999) observaram a formação do olho em larvas de Pagrus pagrus antes do início da alimentação exógena, o que indica que esses animais estavam prontos para a captura de presas. No entanto, apenas aos vinte dias de vida, as primeiras células com núcleo esférico e precursoras da célula bastonete apareceram e aumentaram em tamanho. Esta célula está intimamente relacionada à visão em baixa luminosidade, o que demonstra que, nessa idade, ainda há desenvolvimento morfológico nos sistemas de percepção. Como as larvas de pacu tiveram seu primeiro contato com a dieta microencapsulada somente no momento de sua introdução (após 5 minutos de estímulo), não se pode afirmar que os resultados obtidos foram ocasionados pela aprendizagem das larvas a reconhecer a dieta microencapsulada como alimento, o que certamente influenciaria o resultado final. Cox \& Pankhurst (2000) demonstraram que larvas de Rhombosolea tapirina expostas a uma alimentação composta por rotíferos e Artemia alimentaram-se mais deste microcrustáceo que larvas previamente expostas a uma alimentação exclusiva com rotíferos, o que comprova a existência de aprendizado das larvas em relação às suas presas.

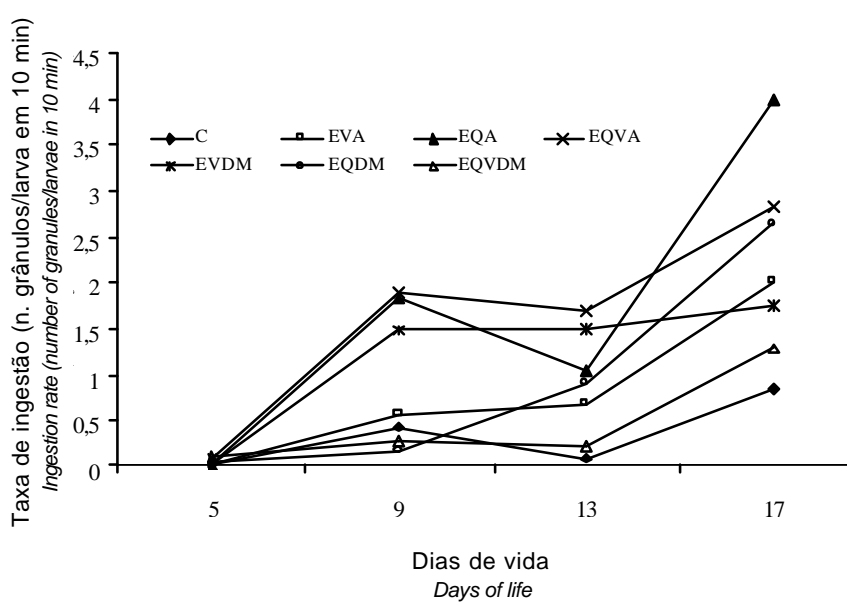

Figura 2 - Valores médios da taxa de ingestão (grãos ingeridos por larva em 10 minutos) para cada estímulo ao longo do experimento.

Figure 2 - Ingestion rate means (number of ingested granules per larvae in 10 minutes) for each stimuli during the experiment. 
O incremento em tamanho, bem como o desenvolvimento das estruturas da cabeça, também são fundamentais para que a larva possa ingerir mais alimento e sustentar seu rápido crescimento contribuindo para o aumento da ingestão. Assim, o aumento da taxa de ingestão com o tempo confirma as observações realizadas por Osse et al. (1997), que correlacionaram o desenvolvimento, o crescimento em comprimento e a necessidade de suprir a demanda de energia para o crescimento da larva. Mesmo assim, isso não invalida os efeitos positivos da estimulação alimentar encontrada neste experimento, pois diferenças significativas $(\mathrm{P}<0,05)$ foram detectadas entre os estímulos testados.

O estímulo químico da Artemia (EQA) e sua combinação com o estímulo visual (EQVA) tiveram maior influência sobre a taxa de ingestão de dieta inerte $(\mathrm{P}<0,05)$ que o tratamento controle e os estímulos visual da Artemia (EVA), químico da dieta microencapsulada (EQDM) e químico + visual da dieta microencapsulada (EQVDM). Resultado intermediário foi obtido com o estímulo visual da ração (EVDM) (Tabela 2). Os resultados indicam ainda que o estímulo químico da Artemia (EQA), em comparação ao estímulo visual da Artemia (EVA), resultou em maiores taxas de ingestão de dieta inerte. Os resultados de Kolkovski et al. (1997) demonstraram que a combinação de ambos os estímulos (visual e químico) resultou em taxas de ingestão superiores às obtidas com as larvas submetidas aos estímulos visual e químico exclusivamente.

Døving \& Knutsen (1993) sugeriram que a atração química das larvas provocada pelos metabólitos secretados por organismos vivos é muito importante para o sucesso da busca e da procura da presa. Rottiers \& Lemm (1985), estudando larvas de Stizostedion vitreum, determinaram que o estímulo químico é essencial para a orientação em direção à presa, enquanto a visão seria mais importante no momento final da apreensão da presa. Desse modo, a combinação de ambos os estímulos (visual e químico) poderia elevar as taxas de ingestão de dietas inertes, fato não observado neste experimento com larvas de pacu.

Diferenças foram encontradas entre os estímulos químico da Artemia (EQA) e químico da ração (EQDM) (Tabela 2). Fatores como a concentração e a composição das substâncias-teste podem explicar a diferença entre estes tratamentos. Shparkovskiy et al. (1983) citam que o comportamento locomotor do salmãoSalmo salarfoi influenciado pelo tipo de aminoácido, do isômero e da concentração.

Independentemente do tratamento, as larvas submetidas a algum tipo de estímulo proveniente dos náuplios de Artemia apresentaram valores superiores de tentativas de captura quando comparadas àquelas sob estímulo proveniente da dieta inerte (Figura 3). As larvas do grupo controle,

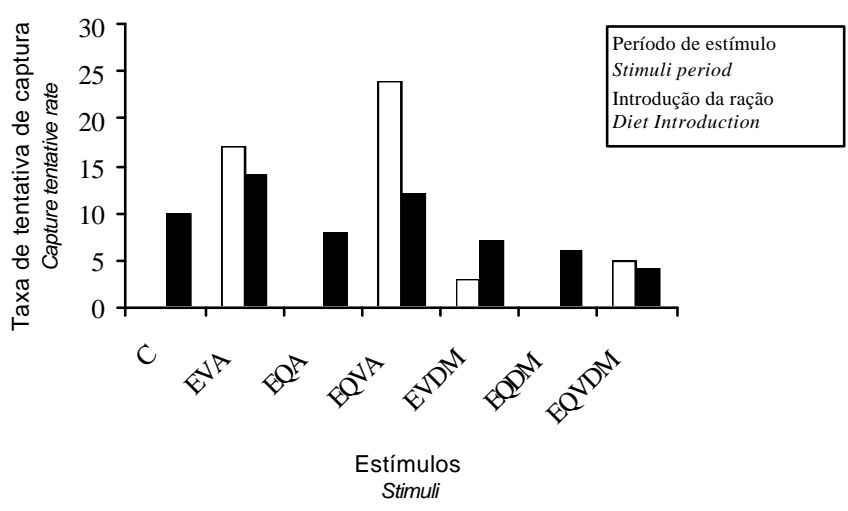

Figura 3 - Valores médios para a taxa de tentativa de captura (número de tentativas de captura/larva/minuto) $(n=3)$ de larvas de pacu submetidas a diferentes estímulos aos 17 dias de vida (12 dias de alimentação exógena).

Figure 3 - Mean values of capture tentative (number of capture tentative/ larvae) ( $n=3)$ of pacu larvae submitted to different stimuli at 1 th day of life (12 days after hatching).

do estímulo químico da Artemia e do estímulo químico da dieta inerte não apresentaram comportamento de captura de alimento. Flütcher (1982) idealizou que alguma substância presente na Artemia, quando utilizada em uma dieta inerte, incrementou o crescimento de larvas de Coregonidae. Kolkovski et al. (1997) determinaram que alguns L-aminoácidos e a betaína excretados pelos náuplios de Artemia tiveram potente efeito estimulador da ingestão de dietas inertes por larvas de Sparus aurata, o que pode auxiliar no desenvolvimento de dietas inertes mais atrativas para as larvas de peixes.

Kolkovski et al. (2000), ao adicionarem a fração solúvel do hidrolizado de krill na água, constataram aumento de $200 \%$ na taxa de ingestão de dieta inerte por larvas de Perca flavescens. Resultado semelhante foi obtido por Harpaz (1997) em camarões gigantes da Malásia (Macrobrachium rosenbergii). Esse autor observou aumento no consumo, com conseqüente acréscimo de $17 \%$ no crescimento, em animais criados com a presença de betaína na água. No entanto, considerando a variedade de substâncias presentes tanto na água de eclosão deArtemia como na água da dieta inerte, qualquer inferência sobre qual substância poderia ter maior efeito estimulante para as larvas de pacu seria mera especulação.

\section{Conclusões}

Os estímulos químico e visual dos náuplios de Artemia e o estímulo visual da ração tiveram efeito positivo 
sobre a taxa de ingestão de dieta inerte por larvas de pacu. O oferecimento de náuplios de Artemia momentos antes do fornecimento de dietas inertes pode auxiliar na transição do alimento vivo para o inerte.

\section{Agradecimento}

À piscicultura São Geraldo, Sertãozinho-SP, pelo fornecimento das larvas de pacu utilizadas neste experimento.

À Coordenação de Aperfeiçoamento de Pessoal de Nível Superior (CAPES), pela concessão da bolsa de estudos.

Ao Conselho Nacional de Desenvolvimento Científico e Tecnológico (CNPq), pelo auxílio financeiro à pesquisa (processo 477812/03-1).

\section{Literatura Citada}

BOCK, C.L.; PADOVANI, C.R. Considerações sobre a reprodução artificial e alevinagem de pacu (Piaractus mesopotamicus, Holmberg, 1887) em viveiros. Acta Scientiarum, v.22, p.495501,2000

CESTAROLLI, M.A.; SALLES, F.A. Ingestão e taxa de alimentação em larvas de peixes tropicais de água doce criadas em laboratório. In: SIMPÓSIO BRASILEIRO DE AQÜICULTURA, 2000, Florianópolis. Anais... Florianópolis: Associação Brasileira de Aqüicultura, 2000 (CD-ROM).

CHABALIN, E.; SENHORINI, J.A.; FERRAZ DE LIMA, J.A. Estimativa do custo de produção de larvas e alevinos. Boletim Técnico do CEPTA, v.2, p.61-74, 1989.

COX, E.S.; PANKHURST, P.M. Feeding behavior of greenback flounder larvae, Rhombosolea tapirina Günther with differing exposure histories to live prey. Aquaculture, v.183, p.285297,2000

DOU, S.; SEIKAI, T.; TSUKAMOTO, K. Feeding behavior of Japanese flounder larvae under laboratory condition. Journal of Fish Biology, v.56, p.654-666, 2000.

DØVING, K.B; KNUTSEN, J.A. Feeding responses and chemotaxis in marine fish larvae. In: Fish nutrition in practice. KAUSHIK, S.J.; LUQUET, P. (Eds.) New York: Chapman \& Hall, 1993. p.579-587.

FLÜTCHER, J. Substance essential for metamorphosis of fish larvae extracted from Artemia. Aquaculture, v.27, p. 83-85, 1982.

GUTHRIE, D.M. Role of vision in fish behavior. In: PITCHER, T.J. (Ed.) The behaviour of Teleost fishes. 2.ed. New York: Chapman \& Hall: London, 1986. p.75-113.

HARPAZ, S. Enhancement of growth in the juvenile freshwater prawns, Macrobrachium rosenbergii, through the use of a chemoattractant. Aquaculture, v.156, p.221-227, 1997.

INSTITUTO BRASILEIRO DO MEIO AMBIENTE E DOS RECURSOS NATURAIS RENOVÁVEIS - IBAMA. Estatística da pesca 2003. Brasil, grandes regiões e unidades da Federação. Brasília: 2004. 89p.
JOMORI, R.K. Estudos sobre a alimentação de larvas de pacu, Piaractus mesopotamicus (Holmberg, 1887) com náuplios de Artemia e sua substituição por dieta artificial Jaboticabal: Universidade Estadual Paulista, 1999. 70p. Monografia (Graduação em Zootecnia) - Universidade Estadual Paulista, 1999.

JOMORI, R.K.; CARNEIRO, D.J.; MALHEIROS, E.B. et al. Growth and survival of pacu Piaractus mesopotamicus (Holmberg, 1887) juveniles reared in ponds or at different initial larviculture periods indoors. Aquaculture, v.221, p.277-287, 2003.

KOLKOVSKI, S.; ARIELI, A.; TANDLER, A. Visual and chemical cues stimulate microdiet ingestion in sea bream larvae. Aquaculture International, v.5, p.527-536, 1997.

KOLKOVSKI, S.; CZESNY, S.; DABROWSKI, K. Use of krill hydrolysate as a feed attractant for fish larvae and juvenile. Journal of the World Aquaculture Society, v.31, p.81-88, 2000.

MUNK, P. Foraging behavior of larval cod (Gadus morhua) influenced by prey density and hunger. Marine Biology, v.122, p.205-212, 1995.

OSSE, J.W.M.; van der BOOGAART, J.G.M.; van SNIK, G.M.J. et al. Priorities during early growth of fish. Aquaculture, v.155, p.249-258, 1997.

PLANAS, M.; CUNHA, I. Larviculture of marine fish: problems and perspectives. Aquaculture, v.177, p.171-190, 1999.

ROO, F.J.; SOCORRO, J.; IZQUIERDO, M.S. et al. Development of red porgy Pagrus pagrus visual system in relation with changes in the digestive tract and larval feeding habits. Aquaculture, v.179, p.499-512, 1999.

ROTTIERS, D.V.; LEMM, C.A. Movement of underyearling walleyes in response to odor and visual cues. The Progressive Fish Culturist, v.47, p.34-41, 1985.

STATISTICAL ANALYSES SYSTEM - SAS. SAS user's guide: statistics. 5.ed. Cary: 1985.

SHPARKOVSKIY, I.A.; PAVLOV, I.D.; CHINARINA, A.D. Behavior of young hatchery-reared Atlantic Salmo salar (Salmonidae) influenced by amino acids. Journal of Ichthyology, v.23, p.140-147, 1983.

SOKAL R.R.; ROHLF, F.J. Biometry. The principles and practice of statistics in biological research. 3.ed. New York: Freeman, 1995. 886p.

SORENSEN, P.W.; CAPRIO, J. Chemoreception. In: EVANS, D.H (Ed.) The physiology of fishes. 2.ed. Florida: CRC, 1997. p.375-405.

TESSER, M.B., CARNEIRO, D.J., PORTELLA, M.C. Co-feeding of pacu (Piaractus mesopotamicus, Holmberg, 1887) larvae with Artemia and microencapsulated diet. Journal of Applied Aquaculture, v.17, p.47-59, 2005. 